Editorial

\title{
Pregnancy in Adolescence - A Challenge Beyond Public Health Policies
}

\section{A gravidez na adolescência - um desafio além das políticas públicas de saúde}

\author{
João Luiz Pinto e Silva ${ }^{1}$ Fernanda Garanhani Surita ${ }^{1}$ \\ ${ }^{1}$ Department of Obstetrics, Faculdade de Ciências Médicas, \\ Universidade Estadual de Campinas, Campinas, SP, Brazil \\ Rev Bras Ginecol Obstet 2017;39:41-43.
}

Recent Sustainable Development Goals were established by the United Nations (2015-2030), including the broad goal of "good health and wellbeing" for all. Good health and wellbeing (item 3 ), quality education (item 4 ), gender equality (item 5) and reduced inequalities (item 10) are among the 17 items of the list. ${ }^{1}$ These four cited goals are directly linked to the unresolved issue of pregnancy during adolescence, and point to fact that well-directed work needs to be done in this area.

According to the United Nations Population Fund (UNFPA), around 16 million girls between the ages of 15 and 19 , and 1 million girls under the age of 15 , give birth every year around the world. In addition, $~ 95 \%$ of these deliveries are concentrated in developing countries, and it is estimated that by 2035 births will be $\sim 20$ million, making pregnancy in adolescence one of the major public health problems. ${ }^{2,3}$ In Brazil, the 2015 National Household Sample Program (PNAD, in the Portuguese acronym) compared the specific fertility rate of women aged 15 to 19 between the years of 2004 and 2014, and this indicator changed from 78.8 to 60.5 children per thousand women in this age group. The participation of adolescents in the total fertility rate remained high, going from 18.4 to $17.4 \%$ in the same period. $^{4}$

Maternity during adolescence draws attention to political debates and issues that have been studied in different areas of knowledge. In the area of public health, teenage pregnancy is considered a major problem due to the high prevalence and perinatal risks.

Adolescent pregnancies may be considered high risk, especially those of adolescents under the age of 15 , and they often fall outside the statistics data. ${ }^{5}$ Childbirth and complications of pregnancy are the leading causes of hospitalization and death among adolescent women in developing countries. Anemia, preeclampsia and postpartum hemor-

Address for correspondence Fernanda G. Surita, MD, PhD, Department of ObGyn, Universidade Estadual de Campinas, R. Alexander Fleming, 101, 13083-881 Campinas, SP, Brazil (e-mail: surita@unicamp.br). rhage may occur in greater proportions than in adult pregnant women. ${ }^{6}$ A recent study among pregnant Brazilian adolescents found that $42 \%$ of them suffered from anemia. ${ }^{7}$ Preterm birth, low birth weight, low Apgar score and perinatal mortality are also more frequent among the children of teenagers. ${ }^{6}$

The nutritional aspects should also be considered a problem in the association of gestation with adolescence, since these are two periods of great metabolic transformations overlapping. Adolescents in general present an accelerated increase in their overall metabolism, and often have poor nutritional habits and make poor food choices, a behavior that generates deficiencies in the intake of some micronutrients, higher rates of overweight and obesity, in addition to a greater frequency of pathological eating disorders, such as bulimia and anorexia. $^{8}$

Obesity may be associated with early menarche, which is one of the risk factors for early pregnancy. During pregnancy, teenagers can experiment excessive gestational weight gain, and that may result in post-pregnancy weight retention and obesity in adult life. Overweightness and obesity affect $\sim 30-40 \%$ of Brazilian women in reproductive age with physical and psychological repercussions leading to an increased risk of non-communicable diseases and a cycle of morbidity and poor health condition in the broadest sense. ${ }^{9-12}$

Besides the aforementioned medical risks, there is an increase in the social risk related to the physical, emotional, economic, and social dependence of these girls. There is also a greater risk of violence, in all its forms, associated with the lack of autonomy of these mothers in making their own decisions. Lack of autonomy can compromise the aspects related to the evolution of the gestation, as well as various aspects of the mother's future life. Examples of this condition include the later onset of prenatal follow-up, ${ }^{6}$ and the higher 
prevalence of "delays" in obstetric care, related to the occurrence of maternal near miss, both of which are higher among adolescents. $^{13}$

Unwanted pregnancy is an important cause of morbidity in adolescence, and the use of effective contraception is one of the fundamental pillars that prevent the occurrence of this problem. ${ }^{14}$ In the United States, teenage pregnancy rates are the highest among high-income countries. ${ }^{15}$ However, there has been a reduction since 1990 , which could be associated with educational programs that address responsible sexuality and contraceptive use among adolescents. Such educational programs would have been responsible for $86 \%$ of this decline. ${ }^{16}$ The use of a "double method" or "double protection," which consists of the use of a condom associated with another effective method, and a more widespread use of long-acting reversible contraceptives (LARCs) accompanied this reduction. $^{17}$

Any reversible method of contraception can be used for adolescents. Neither the age nor the number of previous pregnancies constitutes a medical reason to contraindicate any of them. It is important to remember that, whatever the choice, it should be based on current scientific information, respecting the eligibility criteria of each method established by the World Health Organization (WHO) and the recommendations of the Brazilian Ministry of Health. ${ }^{18,19}$ A Brazilian study shows that after childbirth, the most used contraceptive method was depot medroxyprogesterone acetate (DMPA). To improve contraception and reduce the chance of unintended pregnancies among adolescents, we should promote the use of LARCs. $^{20}$

A recent systematic review of the Cochrane Library compiled studies with a higher quality of information elaboration and consistency, and concluded that it is necessary to integrate educational interventions and offer contraceptive methods in order to reduce the phenomenon of teenage pregnancy. $^{21}$

The abstention from formal education among adolescent pregnant women is relevant, and many of them do not have the opportunity to resume their education once they give birth due to the time they spend in the care of their newborns. $^{22}$ The lower schooling of adolescents at the time of their first pregnancy can be attributed to this serious condition of social deprivation (dropping out of school), and could be one of the reasons behind the etiology of the problem, being the cause, and not the consequence. Disruption of studies in the puerperal period are often unavoidable due to lack of support for these girls and their babies. This all adds to the negative social and economic impact on the adolescents, their families, and society as a whole, for it hinders them from using their potential to invest in their education, find a job and ear an income. The absence from school also increases the chances of a recurring pregnancy still during adolescence. On the other hand, adolescents who do not become pregnant are more likely to finish high school. $^{22}$

In addition to formal education, adolescent sexual education is required. It is widely quoted by the WHO / and The Joint
United Nations Program on HIV/AIDS (UNAIDS) that "Sexual education does not lead to increased sexual activity or stimulation of early sexual activity," and a sexual orientation and education policy is necessary to enable "educated and empowered women and girls to make decisions about their own health," thereby ensuring their reproductive rights, according to Dr. Margaret Chan, Director-General of the WHO.

In this context, the role of the gynecologist-obstetrician is to guide and ensure safe and effective contraception and protection from sexually transmitted diseases. For pregnant teenagers, they could perform prenatal care, preferably with a multidisciplinary team composed of motivated professionals aiming at pregnancy care, as well as health promotion in the general sense. In the puerperal period, it is necessary to pay special attention to contraception, because the risk of another pregnancy is greater then. It is also important to recruit all possible support, so that these young mothers can resume their life plans.

More than an organic look at the risks involved in gestation among adolescents, the meaning of pregnancy varies according to the social context in which the teenager is inserted. The restricted perspective of personal growth by these young women, the lack of educational incentive and labor market, make them find in pregnancy and in the social role of being a mother a life goal. In this sense, it is perceived that dealing with the reality of pregnancy in adolescence involves dealing with contradictions and ethical dilemmas that go beyond the clinical protocols of care.

Adolescent pregnancy requires critical insight. It is a public health problem, and it presents different dimensions as a human and social phenomenon in different cultures, but its occurrence can often disrupt personal growth and development, and jeopardize future opportunities for many girls. Finally, it is a question of gender and vulnerability that reflects all kinds of inequity.

\section{References}

1 United Nations. Sustainable Development Goals [Internet]. New YorkUnited Nations2016[cited 2016 Dec 12]. Available from: http://www.un.org/sustainabledevelopment/sustainabledevelopment-goals/

2 United Nations Population Fund. Universal access to reproductive health: progress and challenges. New York: UNFPA; 2016

3 Fleming N, O'Driscoll T, Becker G, et al; CANPAGO Committee; Society of Obstetricians and Gynaecologists of Canada. Adolescent pregnancy guidelines. J Obstet Gynaecol Can 2015;37(08): 740-759

4 Instituto Brasileiro de Geografia e Estatística [Internet][Synthesis of social indicators: an analysis of the living conditions of the Brazilian population: 2015]. Rio de Janeiro: IBGE; 2015[cited 2016 Apr 10]. Available from: http://biblioteca.ibge.gov.br/visualizacao/livros/liv95011.pdf Portuguese

5 Oliveira FC Jr, Surita FG, Pinto E Silva JL, et al; Brazilian Network for Surveillance of Severe Maternal Morbidity Study Group. Severe maternal morbidity and maternal near miss in the extremes of reproductive age: results from a national cross- sectional multicenter study. BMC Pregnancy Childbirth 2014;14:77

6 Kirbas A, Gulerman HC, Daglar K. Pregnancy in adolescence: is it an obstetrical risk? J Pediatr Adolesc Gynecol 2016;29(04):367-371 
7 Pinho-Pompeu M, Surita FG, Pastore DA, Paulino DS, Pinto E Silva JL. Anemia in pregnant adolescents: impact of treatment on perinatal outcomes. J Matern Fetal Neonatal Med 2016;29:1-5 [Epub ahead of print]

8 Murakami K, Livingstone MB. Decreasing the number of small eating occasions ( $<15 \%$ of total energy intake) regardless of the time of day may be important to improve diet quality but not adiposity: a cross-sectional study in British children and adolescents. Br J Nutr 2016;115(02):332-341

9 Ameade EPK, Garti HA. Age at menarche and factors that influence it: a study among female university students in Tamale, Northern Ghana. PLoS One 2016;11(05):e0155310

10 Yermachenko A, Dvornyk V. Nongenetic determinants of age at menarche: a systematic review. BioMed Res Int 2014;2014:371583

11 Godoy AC, Nascimento SL, Surita FG. A systematic review and metaanalysis of gestational weight gain recommendations and related outcomes in Brazil. Clinics (Sao Paulo) 2015;70(11):758-764

12 Feng Y, Hong X, Wilker E, et al. Effects of age at menarche, reproductive years, and menopause on metabolic risk factors for cardiovascular diseases. Atherosclerosis 2008;196(02):590-597

13 Pacagnella RC, Cecatti JG, Parpinelli MA, et al; Brazilian Network for the Surveillance of Severe Maternal Morbidity study group. Delays in receiving obstetric care and poor maternal outcomes: results from a national multicentre cross-sectional study. BMC Pregnancy Childbirth 2014;14:159
14 Ott MA, Sucato GS; Committee on Adolescence. Contraception for adolescents. Pediatrics 2014;134(04):e1257-e1281

15 Kumar N, Brown JD. Access Barriers to long-acting reversible contraceptives for adolescents. J Adolesc Health 2016;59(03): 248-253

16 Santelli JS, Lindberg LD, Finer LB, Singh S. Explaining recent declines in adolescent pregnancy in the United States: the contribution of abstinence and improved contraceptive use. Am J Public Health 2007;97(01):150-156

17 Eliscu AH, Burstein GR. Updates in contraceptive counseling for adolescents. J Pediatr 2016;175:22-26.e1

18 World Health Organization. Medical eligibility criteria for contraceptive use. 5th ed. Geneva: WHO; 2015

19 Brasil. Ministério da Saúde. Sexual rights, reproductive rights and contraceptive methods. Brasília (DF): Ministério da Saúde; 2009

20 Borovac-Pinheiro A, Surita FG, D'Annibale A, Pacagnella RC, Pinto E Silva JL. Adolescent contraception before and after pregnancychoices and challenges for the future. Rev Bras Ginecol Obstet 2016;38(11):545-551

21 Oringanje C, Meremikwu MM, Eko H, Esu E, Meremikwu A, Ehiri JE. Interventions for preventing unintended pregnancies among adolescents. Cochrane Database Syst Rev 2016;2:CD005215

22 Timur H, Kokanalı MK, Topçu HO, et al. Factors that affect perinatal outcomes of the second pregnancy of adolescents. J Pediatr Adolesc Gynecol 2016;29(01):18-21 